\title{
Highlights of top quark measurements in hadronic final states at ATLAS
}

\author{
Serena Palazzo ${ }^{1,2, \star}$, on behalf of the ATLAS Collaboration \\ ${ }^{1}$ Università della Calabria \\ ${ }^{2}$ INFN Cosenza
}

\begin{abstract}
Measurements of inclusive and differential top quark production cross sections in hadronic final states, including hadronic $\tau$ decays, in proton-proton collisions with the ATLAS detector at the Large Hadron Collider are presented at center-of-mass energies of 7, 8 and $13 \mathrm{TeV}$. The inclusive cross section measurements reach high precision and are compared to other decay modes and the best available theoretical calculations. Differential measurements of the kinematic properties of top quark events are also discussed. These measurements use boosted top quarks, probing our understanding of top quark production in the $\mathrm{TeV}$ regime.
\end{abstract}

\section{Introduction}

The top quark was discovered by both the CDF [1] and D $\emptyset$ [2] collaborations in 1995 at the Tevatron collider. It is the most massive elementary particle known $\left(m_{\mathrm{t}}=173.34 \pm 0.27\right.$ (stat. $) \pm$ 0.71 (syst.) $\mathrm{GeV}$ [3]) and due to its very short lifetime, the top quark decays before hadronizing. This allows to experimentally test the properties of a bare quark. Top quark physics provides a sensitive probe to test the validity of the Standard Model (SM) and a tool to investigate the Higgs boson properties and to potentially discover physics beyond the SM. Some of the recent results achieved by the ATLAS collaboration [4], using data collected at a center-of-mass energy $(\sqrt{s})$ of 7,8 and $13 \mathrm{TeV}$ are here presented. A more complete list of results is available at the ATLAS public top quark results website [5].

\section{Top quark pair production and decay}

At leading order (LO), top quark pairs can be produced via two mechanisms: gluon-gluon fusion and $q \bar{q}$ annihilation. At the Tevatron collider, in $p \bar{p}$ collisions, the dominat process was the $q \bar{q}$ annihilation, whereas, at the LHC, in $p p$ collisions, the dominant production process is the gluon-gluon fusion. The Feynman diagrams of both processes, at LO, are shown in Figure 1.

At next-to-leading order (NLO) the processes are a mixture of quark-gluon (qg) or antiquark-gluon $(\bar{q} \mathrm{~g})$. Some examples of the Feynman diagrams of these NLO processes are shown in Figure 2.

The top quark decays almost exclusively into a $W$ boson and a bottom quark ( $t \rightarrow W^{+}$and $\bar{t} \rightarrow W^{-}$) since the CKM element $V_{t b}$ is close to 1 . The $t \bar{t}$ events are therefore classified according to the decays

^e-mail: serena.palazzo@cern.ch 


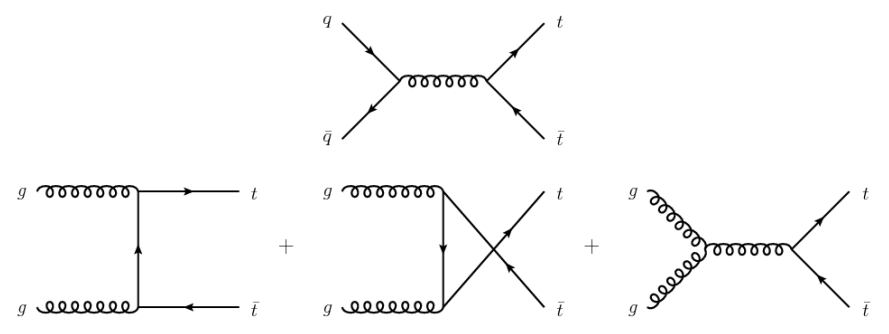

Figure 1. Top pairs production Feynman diagrams at LO. The two production processes are the gluon-gluon fusion and the $q \bar{q}$ annihilation.
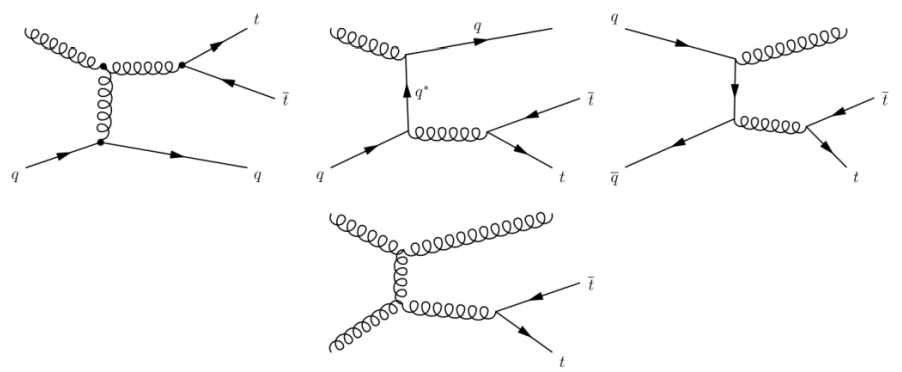

Figure 2. Examples of next-to-leading-order Feynman diagrams for top- antitop pair production via gluon fusion processes and the quark-anti-quark annihilation process.

mode of the two $W$ bosons. When the two $W$ bosons decay hadronically, the event is defined fully hadronic. This is the decay channel with the highest branching ratio $(\approx 44 \%)$ but it has also the largest background. When both the $W$ bosons decay leptonically, the channel decay mode is called di-lepton and it has the lowest branching ratio $(\approx 10 \%)$ but it is the cleanest channel. Then, the last channel is the $\ell+$ jets, characterised by leptonic decay of one of the two $W$ bosons and by the hadronic decay of the second $W$ boson. This channel has a branching ratio of $(\approx 46 \%)$ and it is referred as golden channel because it has a moderate background.

\section{$3 t \bar{t}$ cross-sections in the $\tau+$ jets channel at $\sqrt{s}$ of 7 and $8 \mathrm{TeV}$}

The results of the top quark pairs cross sections in the $\tau+$ jets channel are here presented for the centerof-mass energies of $\sqrt{s}$ of 7 [6] and $8 \mathrm{TeV}$ [7] with an integrated luminosity of $1.67 \mathrm{fb}^{-1}$ and $20.1 \mathrm{fb}^{-1}$, respectively. The final states consist of a hadronically decaying $\tau$ lepton $\left(\tau_{\text {had }}\right)$ and jets corresponding to $t \bar{t} \rightarrow\left[b \tau_{\text {had }} v_{\tau}\right][b q q]$. Such an event topology corresponds to about $10 \%$ of the top quark pairs decays and this measurement is particularly important for charged Higgs boson production in top quark decays. In fact the existence of a charged Higgs boson would lead to an enhancement in the cross section for the considered top quark pair final state.

Starting with the measurement at $7 \mathrm{TeV}$, the candidate events are required to contain at least five jets with a transverse momentum $\left(p_{\mathrm{T}}\right)$ greater than $20 \mathrm{GeV}$. Two of these five jets must be originated from $b$-quarks. Concerning the other three jets, two of them must come from the hadronic decay of one of the two top quarks. The other jet, with high $p_{\mathrm{T}}$, must be selected as $\tau$ candidate. This jet must 
have $p_{\mathrm{T}}$ greater than $40 \mathrm{GeV}$. The majority of $\tau$ decays are characterized by the presence of one or three charged hadrons in the final state. In this analysis, the number of tracks associated to a $\tau$ is used to separate the $\tau$ contribution from the background. The power of this separation is shown in Figure 3 where the distribution of the number of tracks is shown for the $\tau$ component (black line), the electron component (red dotted line) and the multijets events (blue line).

To extract the signal from the distribution of the number of tracks, the data sample is fitted with three probability density functions called also templates. These three templates are the $\tau /$ electron template which is obtained from $t \bar{t}$ simulated events; the remaining significant contributions are due to misidentified jets and are separated into two templates: the gluon-jet which describes the fakes jet coming from QCD multijet processes and the quark-jet template which describes the remaining processes (fakes-jet from $t \bar{t}, W+$ jets and single-top quark events)

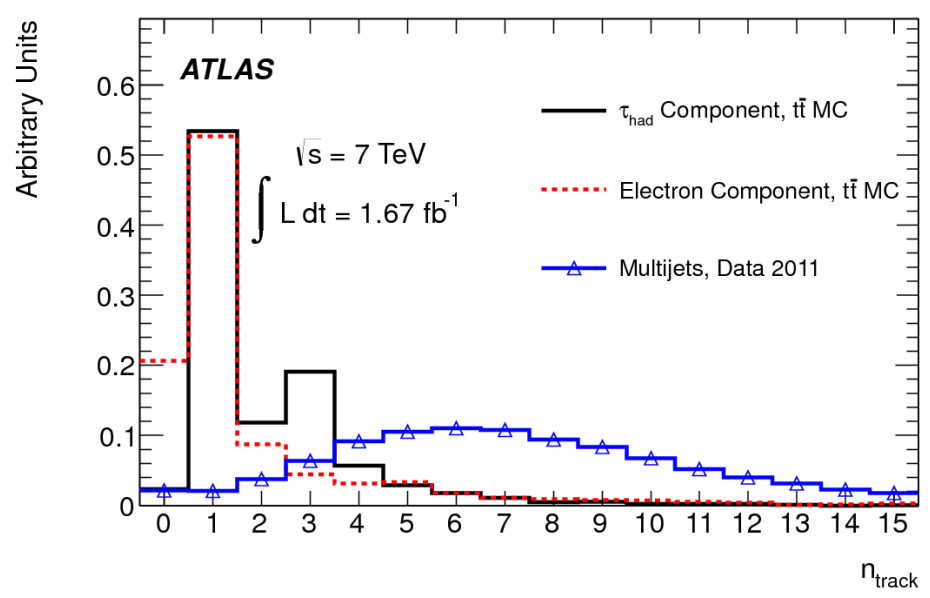

Figure 3. Distribution of the number of tracks for $\tau_{\text {had }}$ from Monte Carlo (MC) $t \bar{t}$ events (solid black line), electrons from MC $\bar{t}$ events (dashed red line), and for jets from multijet events from data (blue triangles) [6].

A likelihood fit is used to extract the different contributions from the distribution of the number of tracks. The fit is shown in Figure 4 where are shown the events as a function of the number of tracks. To extract the number of signal events, predictions from simulation, are used to substract the background of $W+$ jets and single-top quark events from the fitted number of $\tau /$ electron. This number is then scaled by the expected ratio that yield to the number of observed signal events from which the cross section is obtained. In this measurement, the leading uncertainties on the cross section come from the modeling of the initial and final state radiation (about 15\%) and from the choice of the matrix element event generator (about $11 \%$ ). The total systematic uncertainty on the cross section is $24 \%$. Moving to the same analysis but using data collected at a center-of-mass-energy of $8 \mathrm{TeV}$, for this measurement four jets are required. At least two jets with missing transverse energy greater than $25 \mathrm{GeV}$ and two jets originating from $b$-quark. Each event is also required to have at least one $\tau_{\text {had }}$ candidate which dacays into one or three charged particles.

The $\tau$ decays are identified as single prong $\left(\tau_{1}\right)$ when the $\tau$ decays to a single charged particle or three prong $\left(\tau_{3}\right)$ when it decays into three charged particle. To determine the number of signal events, two sources of background are estimated and subtracted: the first source of background containing real $\tau$ in the final state include single-top quark, $W / Z+$ jets and diboson events; the other background 


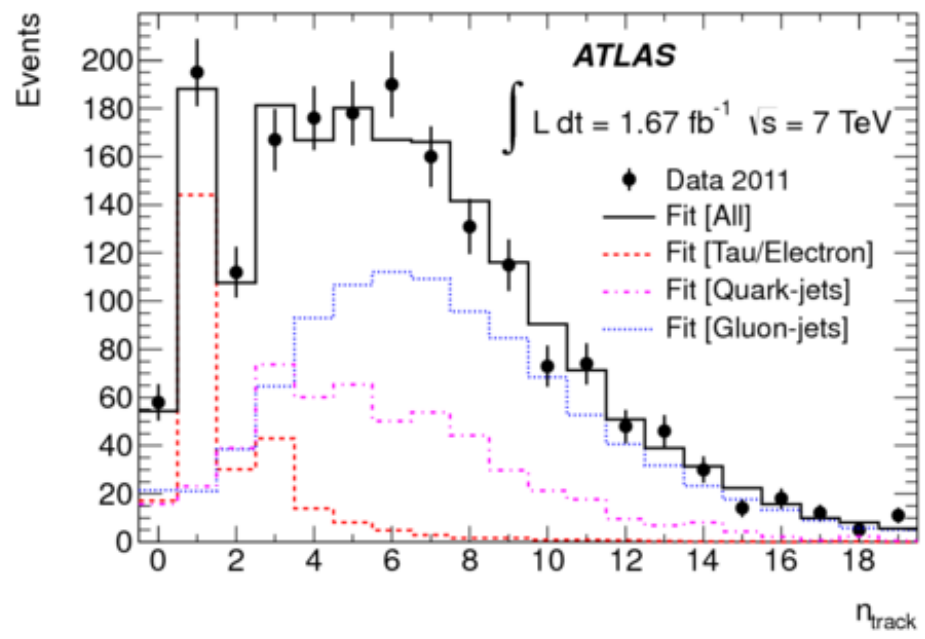

Figure 4. Distribution of the number of tracks for $\tau_{\text {had }}$ candidates after all selection cuts. The black point corresponds to the data while the solid black line is the fit result. Then the red dotted line correspond to the contribution from the $\tau$ /electron signal while the magenta and blue lines correspond to the quark-jet and gluonjet background respectively [6].

source contains events where the $\tau$ in the final state is misidentified and is dominated by multijet processes. In order to determine the $t \bar{t}$ cross section the estimated backgrounds are substracted from the data. In Figure 5 the measured transverse momentum distribution is compared to the background and signal components and a good agreement is observed. The cross section is evaluated for both the $\tau_{\text {had }}$ that decays into one charged particle and for the $\tau_{\text {had }}$ that decays in three charged particles and then, the measurement is combined obtaining the total cross section $\sigma_{t \bar{t}}=239 \pm 4$ (stat.) \pm 28 (syst.) \pm 5 (lumi.) pb. The total uncertainty on the measurement is $12 \%$. 


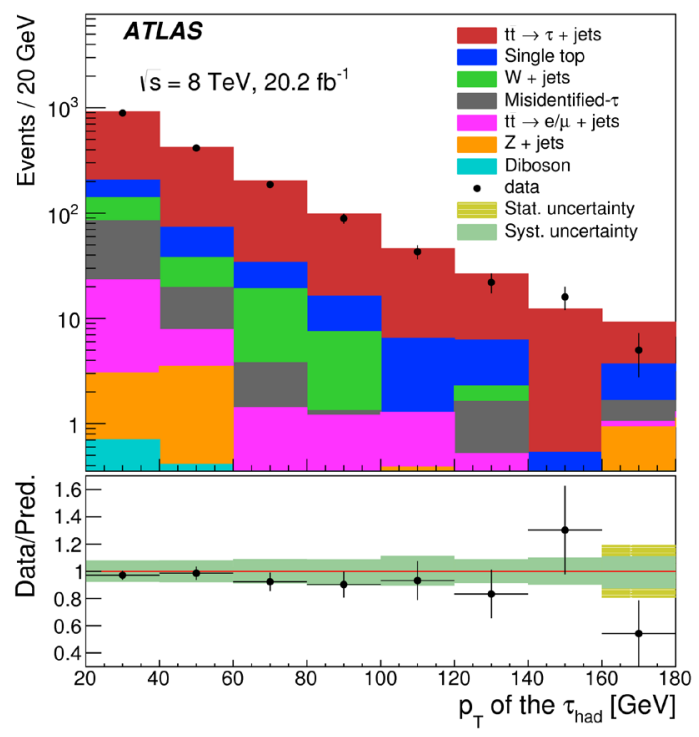

Figure 5. Distribution of the $p_{\mathrm{T}}$ of the $\tau_{\text {had }}$. The measurements are compared to the predictions [7].

\section{$4 \tau+$ lepton $t \bar{t}$ cross-section with $\sqrt{s}=7 \mathrm{TeV}$}

This analysis [8] uses a luminosity of $2.05 \mathrm{fb}^{-1}$. The final state has an electron $(e)$ or a muon $(\mu)$ from the decay of the $W$ bosons and an hadronically decaying of the $\tau$ lepton from the other $W$ boson. This measurement is important because this channel is sensitive to the charged Higgs decays.

The event selection of this analysis is characterized by a primary vertex with at least five tracks, each one with a transverse momentum greater than $4 \mathrm{GeV}$. The additional conditions are: the presence of at least one $\tau$ candidate, only one isolated high $p_{\mathrm{T}} \mu$ or $e$ and a missing transverse energy greater than $30 \mathrm{GeV}$ (to reduce the multijet background).

The $\tau$ identification methodology uses a boosted decision tree discriminants (BDT) to separate hadronic $\tau$ from jets. The BDT distributions could have a lepton and a $\tau$ candidate with the same sign, or a $\tau$ candidate and a lepton with opposite sign.

To estimate the background of this measurement, two different approaches are used. The first approach consists of a fit of the BDT distribution with a background and a signal template. The results of this method is reported on Table 1 . The other method, used as a consistency check, is a matrix method based on a cut on the BDT output. The results of this method are shown in Table 2. As can be seen from the tables, the results obtained using the two methods, are in good agreement within the quoted uncertainties.

The cross section is derived from the difference between the same sign and the opposite sign events in the data sample with at least a $b$-tagged jet. The results are given separately for the single prong and three prong candidate events. The two results are then combined obtaining for the cross section the value $\sigma_{t \bar{t}}=186 \pm 13$ (stat.) \pm 20 (syst.) \pm 7 (lumi.) pb, in agreement with the SM predictions. In Figures 6(a) and 6(b) are reported, respectively, the results of the BDT distributions of $\ell+\tau$ events for the $\tau_{1}$ type and the $\tau_{3}$ type. 
Table 1. Fit results using the background templates derived from $0 b$-tag data and $W+1$ jet. The MC columns give the expected $\tau$ signal, including also the contribution of non- $t \bar{t} \rightarrow \ell+\tau$ events and the expected number of $t \bar{t} \rightarrow \ell+\tau$ events after subtracting the contribution from non- $t \bar{t}$ events to the signal, assuming the theoretical $t \bar{t}$ cross section $(164 \mathrm{pb})[8]$.

\begin{tabular}{llllll}
\hline \hline & \multicolumn{4}{l}{ Background template } & MC \\
& & $0 b$-tag & $W+1$ jet & Signal & $t \bar{t}$ \\
\hline$\mu+\tau$ & $\tau_{1}$ & $490 \pm 40$ & $456 \pm 32$ & 432 & 388 \\
& $\tau_{3}$ & $135 \pm 33$ & $130 \pm 50$ & 126 & 116 \\
\hline$e+\tau$ & $\tau_{1}$ & $440 \pm 50$ & $430 \pm 50$ & 388 & 388 \\
& $\tau_{3}$ & $116 \pm 32$ & $120 \pm 28$ & 114 & 101 \\
\hline Combined & $\tau_{1}$ & $930 \pm 70$ & $860 \pm 50$ & 820 & 726 \\
& $\tau_{3}$ & $260 \pm 60$ & $260 \pm 40$ & 239 & 217 \\
\hline \hline
\end{tabular}

Table 2. Matrix method, based on a cut on the BDT output [8].

\begin{tabular}{llll}
\hline \hline & \multicolumn{3}{c}{ Background template } \\
& & $0 b$-tag & $W$ jet \\
\hline$\mu+\tau$ & $\tau_{1}$ & $460 \pm 60$ & $440 \pm 50$ \\
& $\tau_{3}$ & $130 \pm 40$ & $105 \pm 35$ \\
\hline$e+\tau$ & $\tau_{1}$ & $420 \pm 60$ & $350 \pm 50$ \\
& $\tau_{3}$ & $140 \pm 40$ & $160 \pm 40$ \\
\hline Combined & $\tau_{1}$ & $880 \pm 70$ & $800 \pm 70$ \\
& $\tau_{3}$ & $270 \pm 60$ & $260 \pm 60$ \\
\hline \hline
\end{tabular}

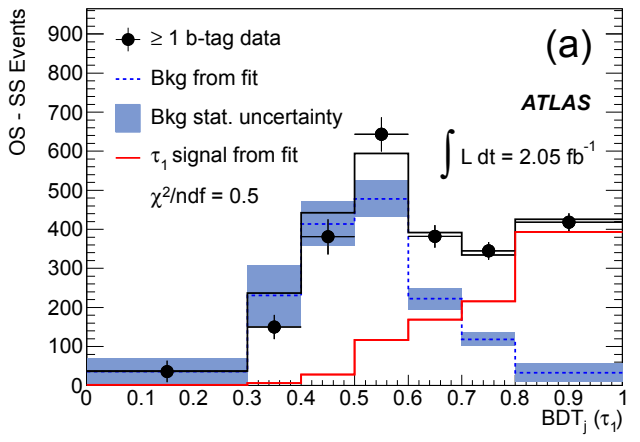

(a)

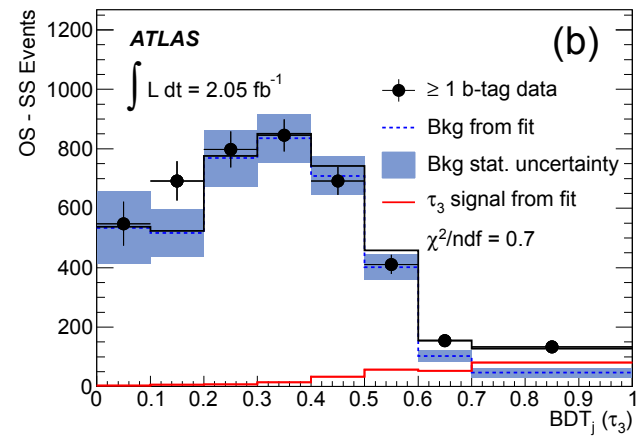

(b)

Figure 6. BDT distributions of $\ell+\tau$ events for the $\tau_{1}$ (a) type and the $\tau_{3}$ (b) type. In the figures the $\tau_{1}$ and $\tau_{3}$ simulations are indicated by the red lines and the background by the dashed blue line. The blue band indicate the statistical uncertainty [8]. 


\section{$5 t \bar{t}$ cross-section in the all-hadronic channel at $\sqrt{s}=7 \mathrm{TeV}$}

This analysis [9] uses $4.7 \mathrm{fb}^{-1}$ of $p p$ collision produced at the LHC and recorded by the ATLAS detector. The final state consists of the hadronically decay of the two $W$ bosons and is characterized by a six-jet topology. This kind of event topology corresponds to $46 \%$ of $t \bar{t}$ decays, it has therefore a large branching ratio but also a large multijet background. This measurement is an important test of perturbative QCD and in addition allow to understand one of the major background to many new physics scenarios.

The events on this measurement are required to have at least a reconstructed primary vertex with five or more associated tracks. All jets are reconstructed with a jet vertex fraction smaller than 0.75 and $p_{\mathrm{T}}$ greater than $20 \mathrm{GeV}$. In addition at least five jets (out of which two should be $b$-tagged) are required with $p_{\mathrm{T}}$ greater than $55 \mathrm{GeV}$ and $|\eta|$ smaller than 2.5 . One more jet is required with $p_{\mathrm{T}}$ greater than $30 \mathrm{GeV}$ and $\eta$ less than 2.5. The dominant uncertainties are jet energy scale, $b$-tagging and and initial and final state radiation.

A kinematic fit has been performed to optimize the top quark mass reconstruction of $\bar{t} \bar{t}$ events. This kinematic fit is based on a likelihood approach. The variable used to perform this fit and extract the cross section is the top quark mass distribution $\left(m_{\mathrm{t}}\right)$. In Figure 7 is shown the fit of this variable to the selected data.

The value of the measured cross-section is $\sigma_{\bar{t}}=168 \pm 12$ (stat.) ${ }_{-57}^{+60}$ (syst.) \pm 7 (lumi.) pb and it is compatible with the SM predictions.

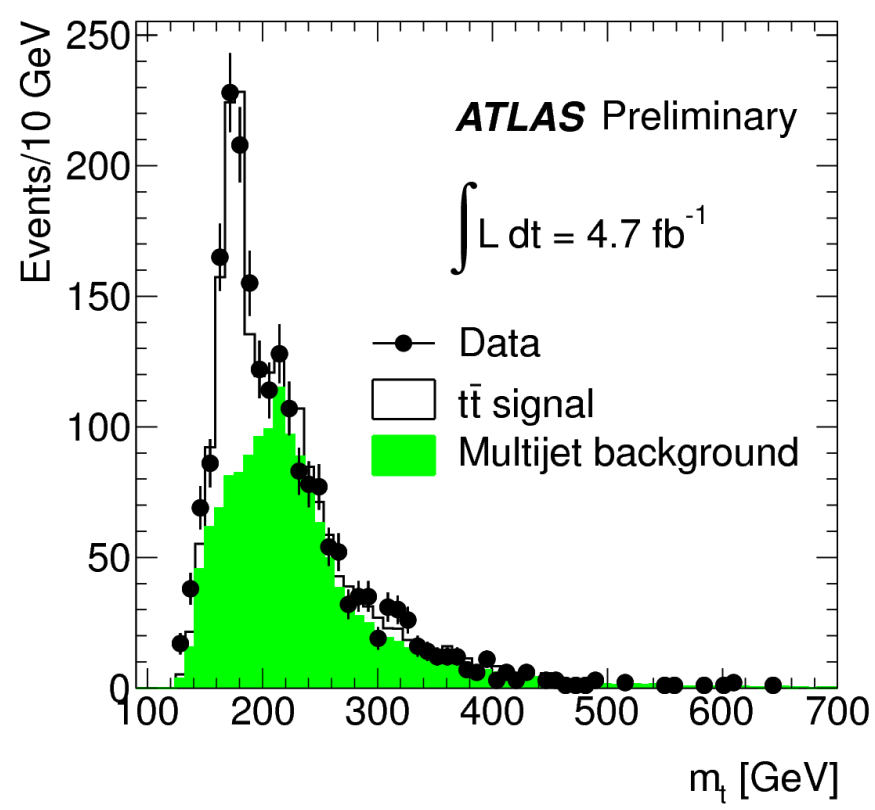

Figure 7. Fit of the $m_{\mathrm{t}}$. The error bars of the data are only statistical. The $t \bar{t}$ signal in white and the multijet background in green are indicated as well [9]. 


\section{$6 t \bar{t}$ differential cross-section in the all-hadronic channel at $\sqrt{s}=13 \mathrm{TeV}$}

This measurement [9] uses $14.7 \mathrm{fb}^{-1}$ of data collected by the ATLAS detector in 2015 and in the first part of the 2016. In this analysis the final state consists of both $W$ bosons decaying hadronically and are selected only top quark candidates with high $p_{\mathrm{T}}$, the selected events contain therefore "fat jets". This measurement allows detailed studies of high $p_{\mathrm{T}} \mathrm{SM}$ processes and searches of anomalies that could be signals of new physics. The event selection require for each event to have a primary vertex with five or more charged tracks. The reconstructed electron or muon should have $p_{\mathrm{T}}$ not greater than $25 \mathrm{GeV}$. The event should have at least 2 large-R jets with $p_{\mathrm{T}}$ greater than $350 \mathrm{GeV}$ and the leading jet should have $p_{\mathrm{T}}$ greater than $500 \mathrm{GeV}$. Events are also required to have at least 2 small-R jets with $p_{\mathrm{T}}$ greater than $25 \mathrm{GeV}$ and 2 small-R $b$-tagged jets each associated with one of the large- $\mathrm{R}$ jets. The largest background source came from multijets events. A data driven approach is employed to estimate the multijet background.

The differential cross section is obtained in a fiducial phase space at particle level using an unfolding tecnique that correct for detector effects. The differential cross sections have been obtained for different distributions. Just some of these distributions are here shown. In Figures 8 the hadronic top variables are shown: Figure 8(a) the normalised differential cross section of the leading $p_{\mathrm{T}}$ and in Figure 8(b) the normalised differential cross section of the second leading $p_{\mathrm{T}}$. In general there is a good agreement between data and prediction within uncertainties.

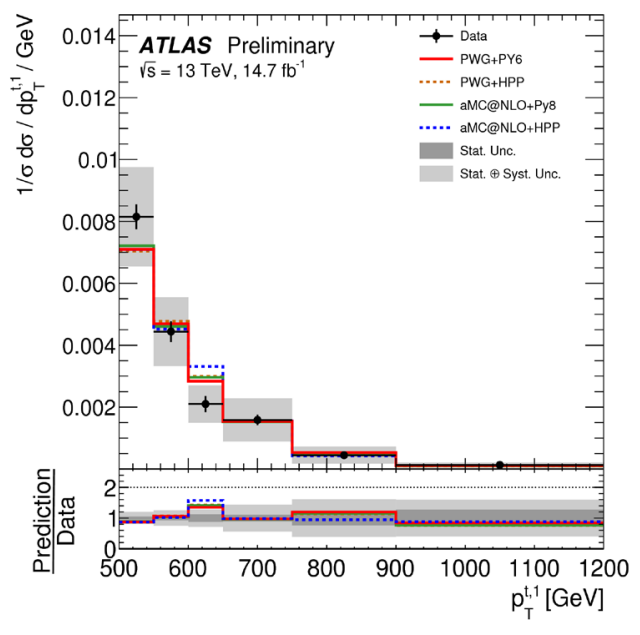

(a)

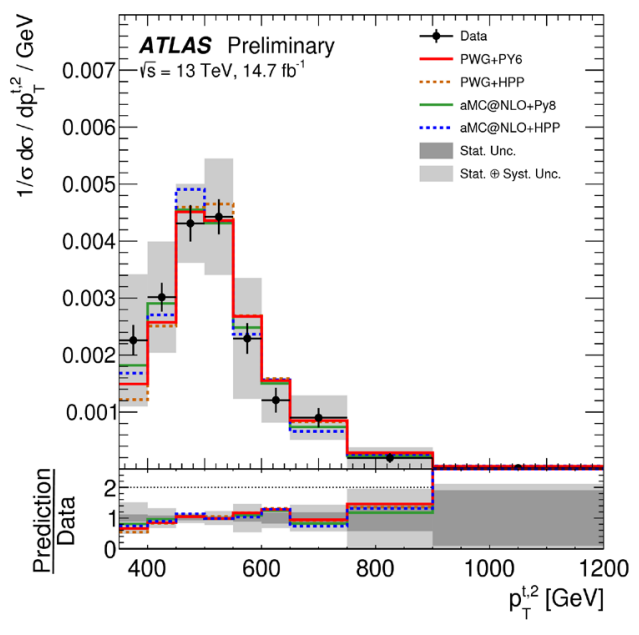

(b)

Figure 8. Normalised differential cross-section for the leading top quark $p_{T}$ in Figure (a) and for the sub-leading top quark $p_{T}$ in Figure (b) [10].

Two differential cross sections as a function of variables of the $t \bar{t}$ system are also shown in Figure 9. In Figure 9(a) the differential cross section for the $t \bar{t}$ system $p_{\mathrm{T}}$ is shown, while, in Figure 9(b) the differential cross section for the $t \bar{t}$ system mass is shown. The two distributions show a good agreement between data and predictions within the total uncertainties. In general the dominant uncertainties components are the large- $\mathrm{R}$ jets, the signal modeling and the $b$-tagging. The cross section measured is $\sigma_{\text {fid }}=374 \pm 13$ (stat.) ${ }_{-92}^{+111}$ (syst.) pb and it is in agreement with the standard model predictions. 


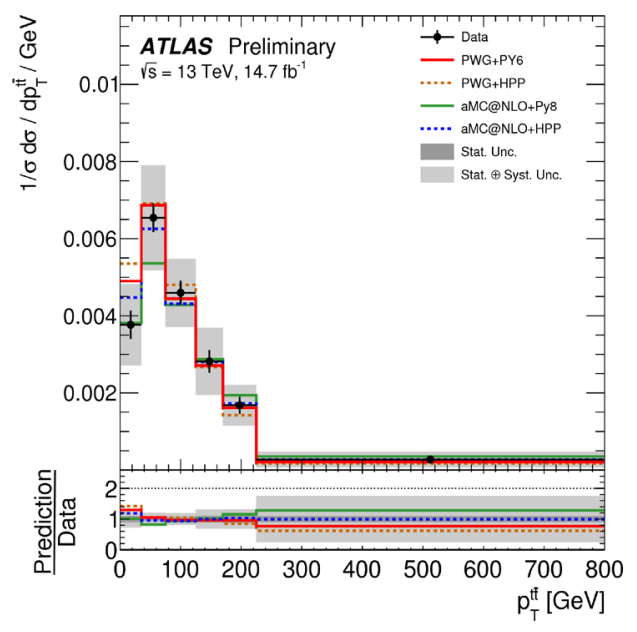

(a)

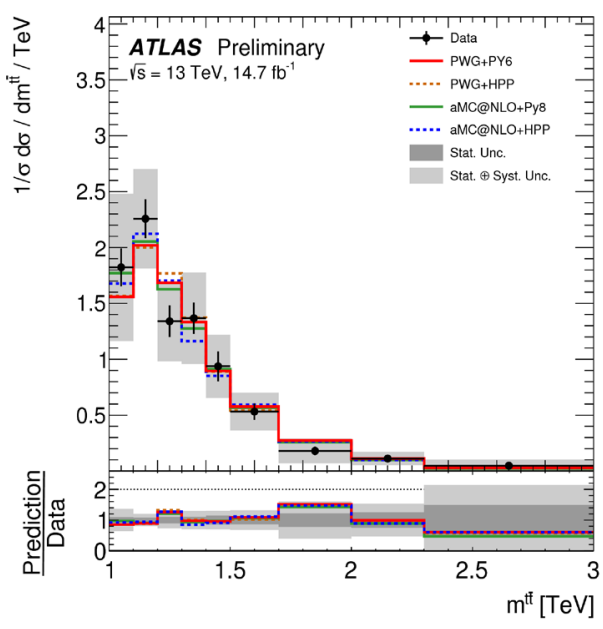

(b)

Figure 9. Normalised differential cross-section for the leading top quark $p_{T}$ in Figure (a) and for the sub-leading top quark $p_{T}$ in Figure (b) [10].

\section{Conclusions}

High precision measurements of top quark inclusive and differential cross sections have been performed by the ATLAS experiment at 7,8 and $13 \mathrm{TeV}$. The results shown are in good agreement with latest SM theory predictions. It has been shown that the ATLAS experiment is testing the SM at high precision with the cross section measurements. All the measurements will benefit from the incoming data samples which will allow to do more precise measurements.

\section{References}

[1] CDF Collaboration, Phys. Rev. Lett 8 (74) 2626 [hep-ex/9503002].

[2] D0 Collaboration, Phys. Rev. Lett. 8 (74) 2632 [hep-ex/9503003].

[3] ATLAS Collaboration, Measurement of the top quark mass in the $t \bar{t} \rightarrow$ dilepton channel from $\sqrt{s}=8 T e V$ ATLAS data, Phys. Lett. B 761 (2016) 350-371.

[4] ATLAS Collaboration, JINST 3 (2008) S08003.

[5] https://twiki.cern.ch/twiki/bin/view/AtlasPublic/TopPublicResults.

[6] ATLAS Collaboration, Measurement of the $t \bar{t}$ production cross section in the $\tau+$ jets channel using the ATLAS detector, Eur.Phys.J. C73 (2013).

[7] ATLAS Collaboration, Measurement of the $t \bar{t}$ production cross section in the $\tau+$ jets final state in $p p$ collisions at $\sqrt{s}=8 \mathrm{TeV}$ using the ATLAS detector, Phys. Rev. D 95, 072003.

[8] ATLAS Collaboration, Measurement of the top quark pair cross section with ATLAS in $p p$ collisions at $\sqrt{s}=7 \mathrm{TeV}$ using final states with an electron or a muon and a hadronically decaying $\tau$ lepton, Phys.Lett. B717 (2012) 89-108. 
[9] ATLAS Collaboration, Measurement of the $t \bar{t}$ production cross section in the all-hadronic channel in ATLAS with $\sqrt{s}=7 \mathrm{TeV}$ data, ATLAS-CONF-2012-031.

[10] ATLAS Collaboration, Measurements of $t \bar{t}$ differential cross-sections in the all-hadronic channel with the ATLAS detector using highly boosted top quarks in $p p$ collisions at $\sqrt{s}=13 \mathrm{TeV}$, ATLASCONF-2016-100. 